\title{
Extensión y derechos humanos: una experiencia de extensión universitaria sobre memoria reciente con organizaciones canarias (2018-2020)
}

\author{
Matías Matta, Nadia Silvestre, ${ }^{2}$ Gonzalo Pintado, ${ }^{3}$ Camilo Argimón ${ }^{4}$
}

Recibido: 30/03/2021; Aceptado: 12/07/2021

DOI: https://doi.org/10.37125/ISR.7.1.5

\section{Resumen}

En los últimos años el departamento de Canelones ha visto florecer una importante cantidad de organizaciones sociales vinculadas a la recuperación de la memoria del pasado reciente, las cuales junto con la Secretaría de Derechos Humanos de la Intendencia de Canelones han desarrollado diversas actividades en todo el territorio canario. Estos colectivos han puesto en marcha diversos proyectos que buscan visibilizar aquellos lugares que fueron testigos de la actuación ilegítima del Estado y el terrorismo de Estado entre 1968 y 1985, así como también aquellos espacios que se convirtieron en lugares de resistencia frente a la dictadura cívico-militar. Los proyectos desarrollados por estas organizaciones se enmarcan en una amplia gama de propuestas que incluyen sitios y circuitos de memoria, charlas, conmemoraciones, talleres, ferias, entre otros; y un importante trabajo con la comunidad en cada una de las localidades donde actúan estos colectivos. Sin embargo, las organizaciones plantean que dichas actividades se enfrentan a un problema de visibilización tanto a nivel departamental como nacional. El artículo presenta el proceso de trabajo de un proyecto de extensión universitaria desarrollado junto a las organizaciones: Comisión por la Memoria del Maestro Julio Castro, Asociación Civil Ágora, Comisión Memorias de la Costa, Comisión Fusilados de Soca, Comisión Memorias de Toledo y Comisión Rescate de la Memoria de Atlántida.

Palabras clave: memoria reciente, Canelones, extensión universitaria.

1 Facultad de Ciencias Sociales (FCS), Universidad de la República (Udelar). matiasmatta83@gmail.com

$2 \quad$ FCS, Udelar. nadiasc.29.02@gmail.com

3 Instituto Escuela Nacional de Bellas Artes (IENBA), Udelar. gonzalo.d.pintado96@gmail.com

4 IENBA, Udelar. camusbbx@gmail.com 


\section{Introducción}

La recuperación de la memoria del pasado reciente es un proceso donde varios actores - entre ellos la academia - aportan desde su visión y sus conocimientos. Este proceso persigue varios objetivos y - a nuestro entender y siguiendo la propuesta de Elizabeth Jelin (2002) - uno de sus principales agentes son las organizaciones sociales vinculadas a la recuperación de la memoria del pasado reciente, mayoritariamente integradas por víctimas directas del terrorismo de Estado (Rico, 2008) o familiares de detenidos desaparecidos o asesinados.

Como define Jelin (2002, p. 9), solo recientemente el paradigma de los derechos humanos se ha ido incorporando en la vida social. Esta incorporación puede pensarse como una irrupción de los reclamos por el respeto a los derechos humanos y un reconocimiento de las violaciones a estos por parte del Estado en el pasado reciente. En Uruguay estos procesos se traducen en las demandas por ese reconocimiento por parte del Estado de su responsabilidad frente a los hechos ocurridos en la dictadura cívico-militar - cuyo ejemplo más saliente es la condena de la Corte Interamericana por el caso Gelman contra Uruguay_, pero también en el reconocimiento, a través de la Ley de Sitios de Memoria Histórica del Pasado Reciente (Uruguay, 2018), de aquellos lugares donde existieron acontecimientos vinculados tanto a violación de los derechos humanos como la resistencia. Algunos de estos lugares por la magnitud de los hechos son lugares emblemáticos a nivel nacional e internacional. Sin embargo, también existen lugares en distintas partes del territorio uruguayo en donde la dictadura dejó marcas que solamente quienes ahí viven o vivían pueden reconocer y recordar, por lo que este trabajo se enmarca en la necesidad de rescate y recuperación de «las memorias». Esta voluntad social de recordar (Waldman, 2006, p. 1) se ve plasmada de manera clara en las organizaciones sociales canarias, que trabajan con la recuperación de la memoria del pasado reciente referida a la violación de los derechos humanos a través de la actuación ilegítima del Estado entre 1968 y 1973, ${ }^{5} \mathrm{y}$ del terrorismo de Estado entre 1973 y $1985 .{ }^{6}$ Los trabajos y proyectos desarrollados por las organizaciones de memoria reciente abarcan una amplia gama de actividades que alcanzan altos grados de visibilidad dentro de cada localidad donde actúan estos

5 Según el artículo quinto de la Ley n. ${ }^{\circ} 18.596$ de Reparación a las Víctimas de la Actuación Ilegítima del Estado, «Se consideran víctimas de la actuación ilegítima del Estado en la República Oriental del Uruguay todas aquellas personas que hayan sufrido violación a su derecho a la vida, a su integridad psicofísica o a su libertad sin intervención del Poder Judicial dentro o fuera del territorio nacional, desde el 13 de junio de 1968 hasta el 26 de junio de 1973, por motivos políticos, ideológicos o gremiales. Dichas violaciones deberán haber sido cometidas por parte de agentes del Estado o de quienes, sin serlo, hubiesen contado con la autorización, apoyo o aquiescencia de agentes del Estado» (Uruguay, 2009).

6 Según el artículo cuarto de la Ley n. ${ }^{\circ} 18.596$, «Se consideran víctimas del terrorismo de Estado en la República Oriental del Uruguay todas aquellas personas que hayan sufrido la violación a su derecho a la vida, a su integridad psicofísica y a su libertad dentro y fuera del territorio nacional, desde el 27 de junio de 1973 hasta el 28 de febrero de 1985, por motivos políticos, ideológicos o gremiales. Dichas violaciones deberán haber sido cometidas por parte de agentes del Estado o de quienes, sin serlo, hubiesen contado con la autorización, apoyo o aquiescencia de los mismos» (Uruguay, 2009). 
colectivos debido al contacto permanente que mantienen con otras organizaciones territoriales y con vecinos y vecinas en general. Sin embargo, esta visibilidad - por diversas razones que exceden los objetivos de este trabajo- se ve drásticamente reducida a nivel departamental y nacional. Con el objetivo de contribuir a reducir esta problemática el proyecto de extensión universitaria que da lugar al presente artículo ${ }^{7}$ se propuso, a partir de un pedido de las organizaciones canarias con las que se trabajó, crear una pieza audiovisual, visibilizar los trabajos realizados por los colectivos de memoria reciente para la instalación de sitios y circuitos de la memoria, los hechos y lugares - que han sido recuperados y quedan por fuera del discurso oficial y de los relatos más conocidos y épicos a nivel nacional-, el trabajo de estas organizaciones en su vínculo con otras organizaciones sociales y personas y, por último, el vínculo de estos colectivos con los organismos institucionales vinculados a la materia, en especial la Secretaría de Derechos Humanos de Canelones.

Teniendo en cuenta estas características, el proyecto que da origen a este artículo se vinculó con las siguientes organizaciones: Comisión por la Memoria del Maestro Julio Castro, Asociación Civil Ágora, Comisión Memorias de la Costa, Comisión Fusilados de Soca, Comisión Memorias de Toledo y Comisión Rescate de la Memoria de Atlántida. El equipo de estudiantes que desarrolló este proyecto se encuentra conformado por una estudiante de la Licenciatura en Sociología y uno de la Licenciatura en Ciencia Política de la Facultad de Ciencias Sociales, los cuales pertenecen a la Comisión por la Memoria del Maestro Julio Castro. Y dos estudiantes de la Facultad de Bellas Artes pertenecientes a la Licenciatura en Lenguajes y Medios Audiovisuales. Esta conformación nos permitió por una parte un conocimiento cercano y también una mirada externa que facilitó objetivar las prácticas de todo el equipo, incluyendo la posibilidad de reflexionar críticamente sobre el quehacer de las propias organizaciones.

En concreto y con respecto a la organización interna los estudiantes con formación en ciencias sociales Matías Matta - estudiante de la Licenciatura en Ciencia Política - y Nadia Silvestre - estudiante de la Licenciatura en Sociología- desarrollaron tareas de coordinación con las organizaciones sociales para los diferentes encuentros y participaron de sus intervenciones en cada localidad. Por otra parte, se encargaron de la elaboración de la pauta para las entrevistas, así como también del desgrabado, el análisis de sus datos, la evaluación del proyecto y la elaboración del informe final. Por último, también colaboraron en la creación del archivo documental, montaje y postproducción. Por su parte los integrantes con formación en la Licenciatura en Lengua y Medios Audiovisuales de la Facultad de Bellas Artes,

7 Proyecto: «Canelones también existe: Relevamiento, sistematización y trabajo conjunto con las organizaciones sociales canarias vinculadas a la recuperación de la memoria del pasado reciente», elaborado por quienes suscriben con la tutoría de la docente del Área de Derechos Humanos del SCEAm Natalia Montealegre a quien agradecemos muy especialmente el acompañamiento y apoyo a lo largo de todo el proceso de trabajo, incluyendo la elaboración del presente artículo. El proyecto fue aprobado en la convocatoria a Proyectos Estudiantiles de Extensión Universitaria 2017-2018, de la Comisión Sectorial de Extensión y Actividades en el Medio (CSEAM) de la Universidad de la República. 
Gonzalo Pintado y Camilo Argimón, desarrollaron tareas de preproducción del material audiovisual, registro audiovisual de entrevistas y acciones de las distintas organizaciones en cada localidad, además del montaje y la posproducción del material audiovisual, así como también colaboraron con el análisis de los datos recabados, la evaluación del proyecto y elaboración del informe final.

\section{Antecedentes y conceptos claves}

Respecto al trabajo sobre la memoria del pasado reciente en el Departamento de Canelones hay algunos antecedentes a destacar por el aporte que significaron para el proceso de trabajo con las organizaciones antes mencionadas. Se trata del libro Otra historia: memorias de resistencia. Mujeres de Las Piedras 1968-1985 (Sapriza, Larrobla, Montealegre y Cherro, 2015), producto de una investigación desarrollada por la Facultad de Humanidades y Ciencias de la Educación de la Udelar y la investigación arqueológica a cargo de Nicolás Gazzán (2018), titulada «Plan de trabajo para la preservación y desarrollo del sitio de memoria Los Vagones». Pueden mencionarse además las siguientes películas y documentales: Voces de Santa Lucía (Pauletti y Oyarzábal, 2016), Secretos de lucha (Hautin y Bidegain, 2007), Huellas (Liceo N. ${ }^{\circ}$ 1, Atlántida, 2009) y Memorias de mujeres: la cárcel de Punta de Rieles (Catilla y Martínez, 2005).

Además de estos aportes, entendemos que es necesario precisar cuatro conceptos claves a lo largo de este proceso de trabajo para abordar las acciones realizadas por las organizaciones sociales canarias que trabajan con la recuperación de la memoria del pasado reciente: 1) nuevos movimientos sociales, 2) acción colectiva, 3) memoria, y 4) espacio social. Con respecto al primer concepto estas organizaciones pueden ser catalogadas dentro de lo que Sidney Tarrow (1997) define como nuevos movimientos sociales. Este autor expresa que «el poder de los movimientos se pone de manifiesto cuando los ciudadanos corrientes unen sus fuerzas para enfrentarse a las élites, a las autoridades y a sus antagonistas sociales» (p. 17). Plantea a su vez que crear, coordinar y mantener la interacción es la contribución específica de los movimientos sociales, los cuales surgen cuando se dan las oportunidades políticas para la intervención de agentes sociales que normalmente carecen de ellas, atrayendo a los individuos a la acción colectiva. Cuando los actores sociales conciertan su acción colectiva en torno a aspiraciones comunes en el marco de una interacción con las autoridades y oponentes, surge el movimiento social, al cual Tarrow (1997) define como «desafíos colectivos planteados por personas que comparten objetivos comunes y solidaridad en una interacción mantenida con las elites, los oponentes y las autoridades» (p. 21). En este sentido, los movimientos sociales dependen de su entorno exterior, especialmente de las oportunidades políticas que se presentan, para la coordinación y mantenimiento de las acciones colectivas. 
Con respecto al concepto de acción colectiva son importantes los aportes de Alberto Melucci (1994) quien considera que

... la acción colectiva es considerada resultado de intenciones, recursos y límites, con una orientación construida por medio de relaciones sociales dentro de un sistema de oportunidades y restricciones. Por lo tanto, no puede ser entendida como el simple efecto de precondiciones estructurales, o de expresiones de valores y creencias (p. 14).

Para luego asegurar que las personas actuando colectivamente construyen su acción mediante intervenciones (más o menos) organizadas. La acción colectiva entonces no deriva de acciones mecánicas producidas por las estructuras sociales, sino que es resultado de orientaciones intencionales desarrolladas a través de relaciones sociales que se dan en un medio ambiente de oportunidades y limitaciones. Maurice Halbwachs (2005) plantea que la memoria debe ser entendida en tanto fenómeno en esencia social y colectivo, es decir, en tanto construcción colectiva sometida a fluctuaciones, y transformaciones. Para este autor toda persona necesita de los recuerdos de otras para recordar. Al ser miembros de un grupo, «pensamos en común algunos aspectos, seguimos en contacto con ese grupo y somos aún capaces de identificarnos con él y de confundir nuestro pasado con el suyo» (p. 3). Es así como ciertos pensamientos, que tienen su origen en el grupo o en sus miembros, las personas los creen propios.

Por otra parte, para Paul Ricoeur (2003), la memoria colectiva

... consiste en el conjunto de huellas dejadas por los acontecimientos que han afectado al curso de la historia de los grupos implicados que tienen la capacidad de poner en escena esos recuerdos comunes con motivo de las fiestas, los ritos y las celebraciones públicas (p. 19).

Una de las características principales de la memoria es que no todo se recuerda, ya que esta es selectiva. Las «huellas» a las que alude Ricoeur (2003), que conforman la memoria, no son una representación exacta de los hechos del pasado, sino una construcción sociocultural, en la cual las personas interpretan y reinterpretan acontecimientos ya ocurridos, vividos o no directamente. Como menciona Ricoeur (2003) «los recuerdos personales están inmersos en narrativas colectivas» (p. 22) y la memoria tiene su raíz en lo concreto, en el gesto, la imagen, el objeto, el espacio. Este espacio cobra a su vez un relevante papel ya que en él se plasman en el presente representaciones simbólicas, rituales, conmemoraciones, que refieren a acontecimientos relacionados con el mismo espacio geográfico en un tiempo diferente. En este sentido se entiende al espacio como medio de inscripción, para dichas representaciones, las cuales modifican la propia espacialidad a lo largo del tiempo.

Las organizaciones canarias de derechos humanos, vinculadas a la recuperación de la memoria del pasado reciente llevan adelante una tarea que posee al menos tres dimensiones; la primera hace referencia al hecho de recordar la actuación ilegítima del Estado y el terrorismo de Estado, al menos entre 1968 y 1985. La segunda se encuentra referida a la generación de vínculos, dentro de cada localidad, con diversos 
actores, organizaciones e instituciones. Y la tercera se refiere a la transformación del espacio social a través de la instalación de sitios y marcas como forma de hacer visible aquellos lugares que guardan una relación directa con el pasado reciente. En este sentido, vale la pena recordar que existen otras formas de intervención por parte de dichas organizaciones como son conmemoraciones, manifestaciones públicas, charlas, debates, registros documentales, y otras actividades performativas.

Con respecto a la primera dimensión, el trabajo de estas organizaciones es vital para mantener el recuerdo de aquellos hechos que significaron grandes heridas para la sociedad uruguaya desde una perspectiva que no implica solo recordar para encontrar culpables, sino también recordar con una mirada puesta en el futuro. En este sentido, Jelin (2002) identifica al cono sur de América Latina como una región donde este vínculo se establece con mucha fuerza al tiempo que afirma que

... aquellos actores que luchan por definir y nombrar lo que tuvo lugar durante períodos de guerra, violencia política o terrorismo de Estado...visualizan su accionar como si fueran pasos necesarios para ayudar a que los horrores del pasado no se vuelvan a repetir (p. 12).

Con respecto a la segunda dimensión, y como también define Jelin (2002), «la memoria tiene un papel altamente significativo como mecanismo cultural para fortalecer el sentido de pertenencia a grupos o comunidades» (p. 9). En este aspecto es de vital importancia el trabajo de las organizaciones sociales mencionadas, ya que estas, a través de diversos mecanismos y formas de intervención delimitan diferentes espacios que sirven como catalizadores de vínculos y potencian el sentido de pertenencia dentro de cada localidad. Estos espacios poseen a su vez distintas características según la localidad que permiten establecer un objetivo común y compartido.

Por último y con respecto a la transformación que llevan adelante estas organizaciones en cada localidad, estas no solo deben ser consideradas como transformaciones físicas, sino también como transformaciones simbólicas, que se enmarcan en un proceso colectivo. Estas transformaciones simbólicas son procesos sociales, políticos y culturales, que no están exentos de contradicciones; por lo que su definición última estuvo precedida de determinados acuerdos y disputas que le dieron contenido. Como definen Jelin y Victoria Langland (2003):

Lo que intentamos comprender no es solamente la multiplicidad de sentidos que diversos actores otorgan a espacios físicos en función de sus memorias, sino los procesos sociales y políticos a través de los cuales estos actores (o sus antecesores) inscribieron los sentidos en esos espacios - o sea, los procesos que llevan a que un «espacio» se convierta en un «lugar»- (p. 2).

Por otra parte, es necesario destacar que estas transformaciones simbólicas se encuentran a su vez en disputa frente a otros actores que buscan establecer otra narrativa sobre el pasado reciente, impulsando lo que podríamos llamar políticas del olvido. Este proceso es analizado por Yamandú Acosta (2008), quien plantea: 
El autoritarismo ha desarrollado una cultura del miedo a través de las prácticas del terrorismo de Estado con capacidad de penetrar al cuerpo de la sociedad en su conjunto, internalizando en [él] [...] los resortes subjetivos funcionales a los mecanismos autoritarios de la dominación, con fuerza tal que perdurarán más allá de la presencia en términos de definición dictatorial del poder autoritario (p. 80).

Para diferenciar esta dicotomía sobre el pasado, Eugenia Allier (2010) establece que existen dos memorias: «la memoria de denuncia de la represión», que busca condenar y denunciar la actuación ilegítima del Estado y el terrorismo de Estado; y «la memoria del elogio», que busca referenciar el pasado reciente con la lucha contra el «terrorismo subversivo», tratando de imponer un discurso del olvido a través de una retórica signada por el miedo. Para esta autora, «la ríspida relación entre historia y memoria conlleva que no exista, ni una memoria, ni un relato histórico, sino una constante batalla por el poder librada por las diversas memorias y los usos políticos del pasado» (p. 260).

\section{Construcción de la demanda: el problema de la visibilidad}

La construcción de la demanda implica una participación activa de la academia y la sociedad civil organizada, en este sentido el intercambio de saberes entre los actores involucrados otorga los insumos necesarios para reconocer tanto las necesidades como sus posibles soluciones. Teniendo en cuenta lo desarrollado hasta el momento entendemos importante señalar que estos procesos deben ser potenciados por la comunidad universitaria y en este sentido creemos que es imprescindible seguir fortaleciendo el vínculo entre la Universidad de la República y las organizaciones sociales de derechos humanos en Canelones. Vinculado a esto, es necesario también profundizar los aportes que viene haciendo la Universidad en el proceso de recuperación de la memoria del pasado reciente en el departamento de Canelones y a nivel nacional. Aportar e impulsar desde la academia el trabajo de dichas organizaciones y las transformaciones que llevan a cabo en cada localidad es una responsabilidad claramente definida en el concepto de extensión universitaria de la Universidad de la República. Una extensión con la cual nosotros deseamos y debemos comprometernos, que debe colaborar desde el conocimiento acumulado para construir procesos de trabajo compartidos, identificando los saberes desde una perspectiva que habilite el diálogo y el trabajo sin jerarquizar un saber sobre los otros, sino buscando los modos de complementarlos y potenciarlos en busca de la creación de nuevos conocimientos.

Teniendo en cuenta la conformación de nuestro equipo, es necesario destacar también que «la intervención para su transformación debe ser necesariamente interdisciplinaria. Todas las disciplinas pueden y deberían estar implicadas en procesos de extensión» (Arocena, et al., 2011, p. 38). En concreto, para la construcción de la demanda se participó de múltiples instancias de intercambio - organizadas entre el equipo del proyecto y las organizaciones - con el fin de identificar aquellas necesidades compartidas por todos los colectivos de memoria participantes. Se debe 
mencionar que la inserción en el trabajo de estos colectivos permitió generar espacios de diálogo, intercambio y análisis para la construcción de soluciones conjuntas a problemáticas concretas. La falta de un material audiovisual, que permitiera dar visibilidad al trabajo de las organizaciones de memoria en Canelones, fue considerada una de las principales carencias que podía ser resuelta por parte del equipo de extensión. En este sentido en todas estas instancias la preocupación central hacía referencia a la reducida capacidad de registrar las diferentes actividades territoriales, así como también a la inexistencia de un material que permitiera mostrar el trabajo de las organizaciones, tanto a nivel nacional como departamental. A partir de los espacios de diálogo generados se pudo concluir que el principal aporte que debíamos realizar era la construcción de un material audiovisual para resolver dichas necesidades.

La inserción en el trabajo que llevan adelante las organizaciones sociales vinculadas a la recuperación de la memoria del pasado reciente en Canelones nos permitió identificar - en diálogo con las personas de las organizaciones y en instancias de análisis compartido- determinadas carencias y construir soluciones conjuntas a partir de esta experiencia de extensión. ${ }^{8}$

\section{Observación participante y grupos de discusión}

El registro audiovisual se conformó a partir de la filmación de diversas actividades territoriales, llevadas a cabo por las organizaciones sociales, así como también por el registro de diversas instancias en que las organizaciones se reunieron para discutir y reflexionar sobre diversos temas. Para esto último se hicieron entrevistas y talleres con el objetivo de conocer y profundizar sobre los conceptos de memoria y sitios de memoria. Dicho de otro modo, para llevar adelante este proyecto se hizo un abordaje cualitativo a través de entrevistas y grupos de discusión, estas instancias fueron registradas en formato audiovisual, siempre con el permiso de todos los participantes. Se entrevistó a integrantes de las organizaciones con el objetivo de obtener información que sirvió para conocer con más profundidad el funcionamiento de cada organización. Los grupos de discusión nos permitieron conocer cuál es el motor de estas organizaciones, y abordar las razones y los objetivos que persiguen, es decir, el porqué y el cómo del trabajo que desarrollan. Este registro nos permitió generar un material audiovisual con mayor contenido narrativo, y aportar así a la futura construcción documental de las acciones y los procesos de transformación que llevan adelante dichas organizaciones. Por otra parte, por medio de la observación participante en el

8 Entendemos el proceso de elaboración de este artículo como el inicio de un proceso de sistematización de este proyecto, lo que permitirá poner en escena las prácticas realizadas para que estas puedan servir como motor de la crítica y reflexión sobre sus carencias y virtudes. La sistematización busca «contribuir de forma decisiva a la recreación y a la construcción de teoría, dinamizando dialécticamente la relación entre el conocimiento teórico ya existente - como expresión de saber acumuladoy los nuevos conocimientos que surgen de las nuevas e inéditas situaciones de las experiencias que vivimos» (Jara, 1994 citado en Berrutti, Dabezies, Barrero, 2012, p. 16). 
Mecanismo de Planificación Departamental en Memoria Reciente de la Secretaría de Derechos Humanos de Canelones fue posible delimitar con mayor claridad el énfasis en los contenidos planteados por nuestros interlocutores. Dicho mecanismo es un espacio abierto por esta secretaría para el encuentro con la sociedad civil organizada entorno a la temática de memoria reciente, y persigue los siguientes objetivos: a) coordinación y planificación de actividades, b) puesta a punto de las actividades que cada organización desarrolla, c) discusión y reflexión sobre temas de memoria reciente y d) construcción de políticas públicas. En palabras de la Secretaría de Derechos Humanos de Canelones lo que se busca es:

Diseñar, transversalizar y coparticipar en políticas públicas que desarrollen la memoria colectiva histórica de nuestro departamento en torno al terrorismo de Estado, la dictadura cívico-militar y sus políticas del terror, las organizaciones y acciones canarias que construyeron la resistencia a estas políticas, los crímenes de Estado y la historia y presente de la lucha por su divulgación pública, su reparación y la construcción de una cultura departamental del «Nunca Más» (Presupuesto quinquenal 2016-2020, Intendencia de Canelones, p. 146).

El mecanismo fue una instancia que se llevó a cabo cuatro veces al año, en la que se articularon acciones, se debatieron temas generales y se presentaron los futuros proyectos para los cuales cada organización solicitó recursos. La participación en ellas nos permitió conocer en profundidad cómo trabajan dichas organizaciones y a partir de ahí pudimos generar un registro de su accionar y de sus características. Como mencionamos antes también se generó material audiovisual a través del registro de las distintas instancias y acciones de las organizaciones en las diferentes localidades. El material se encuentra en proceso de finalización y servirá para dar visibilidad a estas organizaciones sociales territoriales, y, por sobre todo, a las transformaciones que estas generan en cada uno de los lugares. A través de las transformaciones en el espacio social se generan cambios externos, pero también internos en las personas, por lo que creemos que la producción documental juega un rol muy importante en estos procesos. Esta producción no solo presentará datos, sino que pretende mostrar personas e historias, narrar vivencias y generar en el espectador una conexión empática con la temática referida. Para cumplir con los objetivos propuestos se acompañó a 6 organizaciones sociales en actividades, propias y con otras organizaciones, así como también en instancias con la Secretaría de Derechos Humanos de Canelones.

\section{Discusiones a partir del ingreso al campo}

En este apartado expondremos algunas discusiones que se dieron luego de la puesta en marcha de nuestro proyecto, debido a ajustes y modificaciones que se adoptaron con el objetivo de mejorar nuestro trabajo de campo y perfilar nuestra acción en pos de lograr los objetivos definidos junto a las organizaciones canarias. Estos 
intercambios y ajustes los agrupamos en torno a tres aspectos centrales e interrelacionados: 1) los objetivos, 2) la construcción de la demanda y 3) la estrategia de intervención.

\section{Ajustes y discusiones pertinentes en torno a los objetivos}

Los objetivos planteados en la etapa de diseño del proyecto fueron los adecuados, sin embargo, estaban planteados en términos demasiado amplios, por lo que fue necesario reformularlos para que fueran más concretos y específicos. Dicha reformulación nos permitió adoptar una mayor especificidad y compartir de mejor manera nuestros conocimientos y las estrategias de intervención definidas para el proyecto. Por otro lado, se agregó a los objetivos planteados inicialmente un análisis del vínculo y la presencia del Estado con respecto a las organizaciones sociales que forman parte del proyecto. Cabe destacar que estas modificaciones fueron el resultado, por un lado, de reiteradas reuniones del equipo de estudiantes y por otro, de las reuniones de equipo con nuestra tutora.

\section{Ajustes y discusiones pertinentes en torno a la construcción de la demanda}

La construcción de la demanda fue adecuada ya que contó con la participación de los colectivos implicados. Esta construcción se vio facilitada por la militancia social de dos estudiantes en una de las organizaciones que forman parte del grupo de colectivos mencionados. Para la elaboración del proyecto general los colectivos tuvieron una participación marginal sin participar en la redacción de la propuesta, aunque cabe destacar que siempre estuvieron en conocimiento de los procesos que se estaban desarrollando. Sin embargo, a partir de la puesta en marcha del proyecto todas las organizaciones participaron activamente en los procesos e instancias desarrolladas, con importantes aportes en el proceso de construcción de la pieza audiovisual. Cabe mencionar que la participación de dos integrantes del equipo en una de las organizaciones sociales que forma parte del grupo de colectivos que participan del proyecto, permitió conocer de primera mano las principales demandas de las organizaciones. En este sentido la construcción de la demanda desde el inicio en la formulación del proyecto estuvo alineada con las necesidades y expectativas de dichas organizaciones. La construcción de la demanda y sus diferentes dimensiones fueron abordadas en conjunto entre el equipo de estudiantes y la tutora, con el objetivo de problematizar y reflexionar sobre estas cuestiones. En este sentido los aportes de nuestra tutora fueron importantes disparadores de nuestra discusión y reflexión sobre este asunto.

\section{Ajustes y discusiones pertinentes}




\section{en torno a la estrategia de intervención}

Con respecto a este tema, y debido a los tiempos de las organizaciones y a las dificultades para coordinar la concreción de los grupos de discusión, se decidió modificar el cronograma y posponerlos para la segunda mitad del año. Esta modificación nos permitió tener más tiempo para coordinar días, horas y lugares que permitieron la participación de la mayoría de los integrantes de cada colectivo. También fue necesario recortar la cantidad de grupos de discusión debido a los tiempos de coordinación requeridos y a la capacidad de las organizaciones sociales para convocar a todos los integrantes. Cabe mencionar que para dicho recorte se tuvo en cuenta que era necesario mantener la diversidad que poseen las organizaciones sociales entre sí y su presencia en diferentes lugares dentro del territorio canario. Con respecto a las técnicas de intervención fue necesario ofrecer seminarios de formación para abordar las diversas técnicas de entrevista, así como también el abordaje teórico de los grupos de discusión; dichos seminarios constaron de varios encuentros del grupo de estudiantes con nuestra tutora.

Como agregado final se debe mencionar que surgió, en el transcurso de las actividades en el territorio, la necesidad de que el grupo de extensión asesorara a algunos colectivos en el desarrollo de proyectos propios de las organizaciones, lo cual fue una experiencia que puso cara a cara el conocimiento universitario con las necesidades de una parte de nuestra sociedad. Con respecto a las estrategias, estas fueron desarrolladas como se habían planificado, aunque existieron reajustes tanto en el cronograma como en las formas de llevar a cabo de intervención en cada territorio. Esto se debió a que fue necesario ajustarse a los tiempos de las organizaciones sociales, así como también propiciar espacios de formación sobre técnicas de investigación cualitativas, fundamentalmente aquellas que nos habíamos propuesto implementar - entrevistas y los grupos de discusión-. Ambos aspectos nos insumieron un tiempo que no se había tenido en cuenta, previo a las actividades y estrategias planificadas. La intervención a modo de registro audiovisual también fue ajustada, luego de tomar conciencia sobre los espacios y tiempos necesarios a la hora de abordar cada una de las actividades descritas antes.

\section{Resultados y consideraciones finales}

Con respecto a los objetivos propuestos podemos asegurar que el desarrollo del proyecto permitió conocer y relevar un importante número de actividades que llevaron a cabo las organizaciones sociales canarias de memoria reciente durante 2018 , 2019 y 2020, las cuales fueron un importante insumo para la creación de la pieza audiovisual. En este sentido se logró identificar las localidades donde desarrollan sus acciones, las transformaciones que han llevado adelante y los vínculos que poseen con otras organizaciones sociales. Se debe mencionar a su vez que tanto las entrevistas como los grupos de discusión permitieron conocer las razones que impulsan a 
estos colectivos en su accionar, los objetivos que persiguen, los vínculos que mantienen con el Estado y por último cómo entienden estas organizaciones el concepto de sitio de memoria. Todo lo dicho aparece representado en la pieza audiovisual que se encuentra en proceso de finalización, sin embargo, se mencionan a continuación algunos de los principales resultados obtenidos. La metodología utilizada nos permitió comprobar que muchas de las actividades que las organizaciones realizan surgen a partir de la interacción con la Secretaría de Derechos Humanos de Canelones. Los espacios abiertos por el Estado para esta interacción funcionan como catalizadores de acciones que comienzan siendo particulares y pasan a transformarse en colectivas. Como menciona Tarrow (1997), la acción de los movimientos sociales depende de su entorno exterior y de oportunidades políticas que faciliten el trabajo colectivo. En este sentido cobran relevancia los aportes de Melucci (1994) para quien la acción colectiva es resultado de las intenciones, recursos y límites que se construyen a partir de las relaciones que se conforman en el marco de un sistema de oportunidades determinado. Las principales intervenciones de las organizaciones canarias de memoria reciente buscan identificar lugares donde se practicó el terrorismo de Estado, pero también aquellos espacios que funcionaron como lugares de resistencia. La visibilización de estos lugares se establece a partir de la instalación de sitios y circuitos de la memoria que buscan revitalizar lo que Ricoeur (2003) denomina memoria colectiva. Esta memoria se refiere al conjunto de huellas que determinados colectivos son capaces de poner a la vista de la comunidad a partir de intervenciones particulares en un espacio definido. Dichas huellas funcionan como motor y lugar de encuentro para recordar los hechos a través de ritos y celebraciones públicas (Ricoeur, 2003) en cada localidad. Llegado este punto se vuelve relevante mencionar las razones por las cuales se hacen estas intervenciones como pudo ser comprobado a partir de las entrevistas y los grupos de discusión existen dos motivos principales. Por un lado, la no repetición de los hechos ocurridos durante el terrorismo de Estado a través de mantener vivos los recuerdos en cada localidad, y por otro la necesidad manifiesta de que la comunidad reconozca los sucesos como hechos acontecidos en su propio territorio. Como define Jelin (2002), los colectivos que buscan referenciar acontecimientos vinculados a períodos de guerra o terrorismo de Estado en América Latina, se caracterizan por una visualización sobre el futuro que implica no repetir el pasado y por la creación de lazos de pertenencia entre la comunidad y los hechos ocurridos. La participación del equipo en las instancias del Mecanismo de Planificación en Memoria Reciente nos permitió reconocer los procesos a partir de los cuales se presentan las diferentes iniciativas para la acción en cada localidad. En este marco pudimos observar cómo la instalación de sitios y circuitos de la memoria se encuentra precedida por diversos intercambios donde los actores participantes manifiestan sus intenciones y preferencias. La instalación de cada sitio o circuito comienza con una idea original, presentada por el colectivo que pertenece a la localidad donde será instalado. Sin embargo, existen una serie de interacciones que sirven para colectivizar y mejorar la idea original. Como mencionan Jelin y Langland (2003), la conversión de los espacios físicos 
en lugares de memoria se encuentra precedida por procesos sociales y políticos que le imprimen un sentido. Como se mencionó antes, las entrevistas y la observación participante nos permitió reconocer estas particularidades al tiempo que el registro audiovisual de las instancias mencionadas nos proporcionó el material para que estas particularidades pudieran ser el núcleo central de nuestra pieza audiovisual.

Podemos asegurar que el desarrollo del proyecto permitió dar respuesta a la necesidad de contar con un material audiovisual que cumpliera con los objetivos surgidos a partir de la construcción de la demanda. Es necesario destacar que al momento de redactar el presente artículo nos encontramos en el proceso de finalización de la pieza audiovisual que servirá para la visibilización de las organizaciones de memoria reciente y sus trabajos dentro del departamento de Canelones. Como forma de mejorar este proceso se debe mencionar que se concluyó con una primera etapa de preproducción, que incluyó la presentación de un avance de la pieza audiovisual con el objetivo de recoger las opiniones de los diferentes colectivos. En este sentido todas las organizaciones realizaron una valoración altamente positiva en términos del objetivo inicial propuesto, todos los colectivos entendieron que la pieza audiovisual cumplía con sus expectativas. Sin embargo, cada organización propuso una serie de ajustes para su caso concreto en la pieza audiovisual en términos de ponderar algunas actividades sobre otras. Todos estos aportes serán tenidos en cuenta para la pieza final producida.

Con respecto al vínculo construido con las organizaciones sociales podemos decir que se alcanzaron altos niveles de confianza entre ambas partes y se establecieron dinámicas colectivas que permitieron un permanente intercambio de saberes. Es necesario destacar que este aspecto es de suma importancia debido a las características particulares que posee la temática general en la que se encontraba inmerso nuestro proyecto. Otro resultado relevante para la construcción de la pieza audiovisual fue la posibilidad de participar de los espacios que la Secretaría de Derechos Humanos de Canelones había establecido para vincularse con las organizaciones de memoria. Esto nos permitió registrar a las organizaciones en un espacio de discusión y reflexión con el Estado como interlocutor. Sin embargo, se debe mencionar que existen dos organizaciones de memoria con las cuales no pudo desarrollarse un vínculo fluido por diversos motivos; en un caso, porque una de ellas no está trabajando de forma prioritaria en este momento el tema de memoria reciente y, en otro, porque la organización está desarrollando actividades esporádicas. Las organizaciones mencionadas no fueron tenidas en cuenta para el desarrollo del presente proyecto.

Por último, podemos asegurar que la experiencia desarrollada a partir del proyecto de extensión que da lugar al presente artículo posee algunas particularidades que vale la pena destacar. La participación de algunos integrantes del equipo en una organización de memoria que formó parte del proyecto permitió un mejor acercamiento tanto a la temática como al resto de las organizaciones; alcanzando altos niveles de intercambio entre academia y sociedad civil organizada. Es necesario destacar en 
este punto que gracias a esta cercanía las entrevistas pudieron realizarse en un marco de confianza que nos permitió abordar de la mejor manera posible una temática tan compleja. En este sentido se puede afirmar también que este vínculo nos aseguró una acertada identificación de las demandas de estos colectivos, así como también un intercambio fluido de saberes que permitió la concreción de los objetivos planteados al inicio. Por otra parte, esta cercanía con las organizaciones nos dio la posibilidad de coordinar y organizar de manera efectiva las intervenciones de nuestro equipo en cada localidad; asegurándonos así registros visuales de alta calidad.

Creemos que la experiencia descrita fue un aprendizaje permanente, tanto para nosotros como para las organizaciones de memoria, que nos brindó la oportunidad de establecer altos grados de intercambio de saber y desarrollar una experiencia de extensión universitaria en su sentido más amplio.

\section{Referencias}

Acosta, Y. (2008). Uruguay 1985-2005. Dimensiones y tensiones de la democracia. Revista Encuentros Uruguayos, (1), 23-33. Recuperado de http://www.encuru.fhuce.edu.uy/images/revistas/revista-encuentros-uruguayos-2008-diciembre.pdf.

Allier, E. (2010). Batallas por la memoria. Los usos políticos del pasado reciente en Uruguay. MontevideoCiudad de México: Ediciones Trilce-unam.

Arocena, R., Tommasino, H., Rodríguez, N., Satz, J., Álvarez, E., y Romano, A. (2011). Cuadernos de Extensión, 1: Integralidad: tensiones y perspectivas. Montevideo: SCEAM, Universidad de la República. Recuperado de https://www.extension.udelar.edu.uy/wp-content/uploads/2017/11/ Cuaderno-n\%C2\%Bo1-integralidad.pdf.

Berrutti, L., Dabezies, M. J., y Barrero, G. (2012). Apuntes para la acción II. Montevideo: sceam, Universidad de la República.

Catilla, R. (Productora), y Martínez, V. (Directora). (2005). Memorias de mujeres: la cárcel de Punta de Rieles [Película]. Uruguay: Buen Cine Producciones.

Gazzán, N. (2018). Plan de trabajo para la preservación y desarrollo del sitio de memoria Los Vagones. Recuperado de http://procurement-notices.undp.org/view_file.cfm?doc_id=158897.

Halbwachs, M. (2005). Memoria individual y memoria colectiva. En La mémoire collective (Trad. P. Gianera). Recuperado de https://revistas.unc.edu.ar/index.php/restudios/article/ view/13479/13665.

Hautin, J. F. (Productor), y Bidegain, M. (Directora). (2007). Secretos de lucha [Película]. Francia: smac.

Jelin, E. (2002). Los trabajos de la memoria. Madrid: Siglo Veintiuno Editores.

Jelin, E., y Langland, V. (Comps.) (2003). Monumentos, memoriales y marcas territoriales. Madrid: Siglo Veintiuno Editores.

Liceo N. ${ }^{\circ}$ Atlántida. (2009). Huellas [Película]. Uruguay. Recuperado de https://www.youtube.com/ watch?v=G8P9_KaMSYE.

Melucci, A. (1994). Asumir un compromiso: identidad y movilización en los movimientos sociales. Zona Abierta, (69), 153-180. 
Pauletti, D. (Productora), y Oyarzábal, M. (Director). (2016). Voces de Santa Lucía [Película]. Uruguay: Caminito Films.

Presupuesto Quinquenal, Intendencia de Canelones 2016-2020 (tomo II). Recuperado de https://www. imcanelones.gub.uy/sites/default/files/pagina_sitio/archivos_adjuntos/tomo_ii_direcciones.pdf

Rico, A. (2008). Investigación histórica sobre la dictadura y el terrorismo de Estado en Uruguay (1973-1985). Montevideo: Universidad de la República.

Ricoeur, P. (2003). La memoria, la historia, el olvido. Madrid: Editorial Trotta.

Sapriza, G., Larrobla, F., Montealegre, N., y Cherro, V. (2015). Otra historia: Memorias de resistencia. Mujeres de las Piedras 1968-1985. Montevideo: FHCE, Universidad de la República.

TARrow, S. (1997). El poder en movimiento. Los movimientos sociales, la acción colectiva, y la política. Madrid: Alianza Editorial.

Uruguay (2009, setiembre 18). Ley n. ${ }^{\circ}$ 18.596: Reparación a las Víctimas de la Actuación Ilegítima del Estado en el Período Comprendido entre el 13 de junio de 1968 y el 28 de febrero de 1985. Recuperado de https://www.impo.com.uy/bases/leyes/18596-2009/11.

Uruguay (2018, julio 13). Ley n. ${ }^{\circ}$ 19.641: Declaración y Creación de Sitios de Memoria Histórica del Pasado Reciente. Recuperado de http://www.impo.com.uy/bases/leyes/19641-2018.

WALDMAn, G. (2006). La «cultura de la memoria»: problemas y reflexiones. Politica y cultura, (26), 11-34. Recuperado de http://www.scielo.org.mx/scielo. php?script=sci_arttext\&pid=So188-77422006000200002\&lng=es\&nrm=iso\&tlng=es. 